\title{
El sesgo mediocéntrico del framing en España: una revisión crítica de la aplicación de la teoría del encuadre en los estudios de comunicación
}

\section{Bitartekoa erdigune izateak framing-ean eragindako alborapena Espainian: komunikazio-ikasketetan enkoadraketaren teoria aplikatzeko moduaren azterketa kritikoa}

\section{The 'media bias' in the Spanish framing literature: a critical review of how communication studies apply framing theory ${ }^{1}$}

\section{Lidia Valera Ordaz ${ }^{2}$}

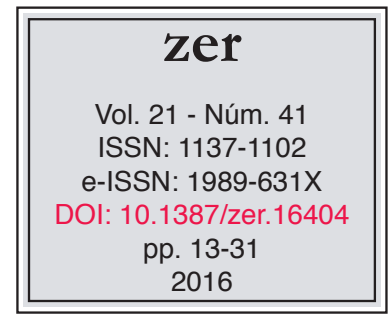

Recibido el 15 de abril de 2016, aceptado el 21 de junio de 2016.

\section{Resumen}

En este artículo se propone una revisión crítica de los enfoques teóricos metodológicos predominantes en la literatura sobre framing en España. Su propósito es señalar que existe un sesgo mediocéntrico, pues los estudios tienden a tomar los encuadres periodísticos como variable independiente, pero no suelen abordan el proceso dinámico y conflictivo de su producción y negociación. Este sesgo responde a una concepción de los marcos como resultado de las rutinas periodísticas, lo que implica desatender las múltiples influencias políticas, sociales e ideológicas que subyacen a la producción de los marcos periodísticos.

Palabras clave: Encuadre, encuadres noticiosos, teoría del framing, discurso, ideología.

\footnotetext{
1 Una versión preliminar de este trabajo fue presentada en el Congreso Iberoamericano "Comunicación, cultura y cooperación" organizado por la AE-IC y celebrado en Madrid entre el 4 y el 8 de julio de 2016. Este artículo ha sido realizado gracias a la beca postdoctoral Vali+D de la Generalitat Valenciana.

2 Universidad de Valencia, lidia.valera @uv.com
} 


\title{
Laburpena
}

Artikulu honetan kritikoki aztertuko dira Espainian framing-ari buruzko literaturan gailentzen diren ikuspegi teoriko eta metodologikoak. Azterketaren helburua da azpimarratzea bitartekoa erdigune duen joera bat dagoela; izan ere, ikasketetan aldagai independente gisa landu ohi dira kazetaritza-enkoadraketak, baina ez dira horiek ekoiztean eta negoziatzean dagoen dinamika- eta gatazka-prozesua jorratzen. Joera horren arrazoia da markoak kazetaritza-errutinen emaitza gisa hartzen direla, eta, hori dela eta, ez da kontuan hartzen kazetaritza-marko horien ekoizpenen atzean hainbat eragin politiko, sozial eta ideologiko daudela.

Gako-hitzak: Enkoadraketa, berrien enkuadraketa, framing teoria, diskurtsoa, ideologia.

\begin{abstract}
This article provides a critical review of the theoretical and methodological aroaches that have prevailed in the Spanish literature on framing. It points out that most studies take news frames as independent variable, but they do not account for the process by which news frames are intentionally built and promoted by stakeholders. We contend that this media bias is the result of a conception of frames as the outcome of journalistic routines, which implies overlooking the social interactions and ideological implications involved in the production and negotiation of frames.
\end{abstract}

Keywords: Frame, news frames, framing theory, ideology, discourse. 


\section{Introducción: el framing, un vasto campo de estudio}

Desde hace décadas, el framing constituye una sólida perspectiva de investigación en los estudios de comunicación fuera y dentro de España. En efecto, la teoría del encuadre ofrece actualmente un aparataje teórico metodológico de referencia en el seno de la ciencia de la comunicación social, que además ha dado lugar a un volumen creciente de producción científica (Vicente y López Rabadán, 2009; ArdévolAbreu, 2015).

Pese a su notable expansión, esta perspectiva exhibe una notoria resistencia a ser definida conceptualmente con la claridad y precisión que exige cualquier paradigma de investigación científica; lo que se traduce en una extraordinaria fragmentación y en no pocos ni banales debates teóricos y metodológicos (Sádaba et al., 2012; Miceviciute, 2013).

Desde un punto de vista específicamente comunicativo, el framing se ocupa de analizar la forma en que se presentan los problemas sociales en los contenidos mediáticos, la interpretación que los medios promueven sobre los asuntos que tratan (Scheufele y Tewksbury, 2007) y el estudio de los mecanismos de selección, énfasis, elaboración y exclusión que subyacen a la producción de contenidos mediáticos (Tankard et al., 1999).

En un sentido general, y retomando sus brillantes raíces sociológicas (presentes en los trabajos pioneros de Gitlin o Tuchman), podemos concebir el framing como un área de investigación que se ocupa de estudiar el proceso de negociación y construcción del discurso público en torno a los asuntos de interés general, y que se despliega principal pero no exclusivamente en el terreno mediático (Pan y Kosicki, 2001; Hertog y McLeod, 2001).

"Ideally, framing research examines how frames are sponsored by political actors, how journalists employ frames in the construction of news stories, how these stories articulate frames, and how audience members interpret these frames" (Carragee y Roefs, 2004: 215). Es decir, el framing aplicado a los estudios de comunicación pone el foco analítico en el discurso mediático, pero sin perder de vista que los medios constituyen un actor esencial en el proceso de producción y construcción del discurso público, pero ni mucho menos el único (Gamson, 1992; Gamson y Modigliani, 1989; Pan y Kosicki, 1993; 2001). La labor de los medios está mediada, en primer lugar, por los actores sociales que trasladan sus producciones simbólicas a la arena mediática y, en segundo, por las interpretaciones que realiza el público de los mensajes.

En este sentido, diversos autores han denunciado el creciente divorcio de la investigación internacional sobre framing de sus primeras raíces sociológicas. Reclaman la necesidad de atender los contextos sociales y políticos más amplios en los que se producen los marcos mediáticos, prestar atención a los aspectos relacionados con la distribución del poder, y concebir el framing como un proceso dinámico, intencional y competitivo, desplegado por actores sociales cuyo objetivo es construir y difundir significados en el espacio público (Carragee \& Roefs, 2004; Maher, 2001; Vliegenthart \& van Zoonen, 2011; Borah, 2011). Reivindican que la mayoría de estudios se ocupan de identificar los marcos que dominan los contenidos mediáticos, pero desatienden las interacciones sociales a través de las cuales esos marcos han sido construidos y negociados. 


\section{Orígenes: de la multidisciplinariedad al anclaje sociológico}

La teoría del encuadre presenta unos orígenes multidisciplinares sobradamente conocidos: procede de diversas disciplinas que se han interesado por analizar cómo la forma de los mensajes comunicativos tiene repercusiones sobre las cogniciones y conductas de los individuos (Sádaba, 2001; Scheufele y Tewksbury, 2007; Vicente y López Rabadán, 2009).

A este respecto, la indagación de los frames hunde sus raíces en la psicología cognitiva (Bateson, 1972), la sociología (Goffman, 1974), la economía, (Kahneman y Tversky, 1979), y, más posteriormente, en la lingüística cognitiva ${ }^{3}$ (Lakoff, 2004). La mayoría atribuye la primera utilización de 'frame' a Gregory Bateson, quien entendía los marcos como principios organizadores de la percepción cognitiva. Pero será la sociología de Goffman (1986) la que concebirá los frames como principios organizativos del mundo social, como estructuras compartidas que dotan de sentido los acontecimientos sociales.

Y es a partir de este anclaje sociológico cuando el framing es importado al estudio de la comunicación de masas, permitiendo a los investigadores interrogarse sobre los marcos que estructuran los mensajes periodísticos y cómo éstos contribuyen a la construcción de significados sociales compartidos. Merece la pena insistir, por lo tanto, en que es la sociología la que canaliza la importación del framing al estudio de la comunicación de masas, situando el análisis de los contenidos mediáticos en el más amplio campo de la producción de discursos sociales dominantes. Así, Gitlin (1980: 7) concibe los marcos como dispositivos a través de los cuales "quienes manejan los símbolos organizan de forma rutinaria el discurso".

Esta fuerte multidisciplinariedad de origen se ha traducido en un enorme abanico de enfoques, de tal forma que en esta perspectiva se han dado cita tanto aproximaciones neopositivistas, basadas en la medición cuantitativa de regularidades empíricas, como ejemplifica el trabajo de McCombs y sus seguidores (Ghanem, 1997; Takeshita, 1997; McCombs, 2004); como enfoques constructivistas, afanados por identificar frames acudiendo al contexto cultural y recurriendo a métodos cualitativos para desentrañar los procesos de problematización de la realidad, entre los que destaca la escuela de William Gamson (Gamson y Modigliani, 1989; Gamson, 1992; Pan y Kosicki, 2001; Van Gorp, 2007).

Así, esta pluralidad ha sido considerada por algunos como una fuente de enorme riqueza analítica (D'Angelo, 2002), capaz de abordar el estudio del discurso mediático a través de una vasta pluralidad teórica y metodológica, pero también como una "maldición" (Hertog y McLeod, 2001: 40), a tenor de la notable ambigüedad e indefinición conceptual que reviste el término frame, de cuyo significado dan cuenta innumerables definiciones, casi tantas como investigadores afanados en su identificación y medición (Entman, 1993; Sádaba, 2001; Reese, 2001; Carragee y Roefs, 2004; Weaver, 2007).

\footnotetext{
El trabajo de Sádaba (2001) ofrece una exposición detallada del origen del concepto, mientras que el Miceviciute (2013) proporciona una interesante discusión sobre la confusión terminológica en torno al término frame, así como sobre su utilización desde distintas tradiciones (psicología, sociología y lingüística). Nosotros emplearemos, no obstante, el término frame como sinónimo de marco y encuadre.
} 
De esta vaguedad conceptual de la noción de frame se derivan numerosas controversias teóricas y metodológicas, significativas dificultades para formular hipótesis contrastables empíricamente, problemas de operacionalización, y, en definitiva, una palmaria falta de valor predictivo que sí exhiben las teorías de rango medio (Maher, 2001). "[The study of] 'frame' can be alied to many different aspects of messages and to many different types of messages. It can also be studied by means of systematic content analysis or more interpretative textual analysis alone" (Weaver, 2007: 144).

Entre la cantidad innumerable de definiciones que existen sobre los frames, merece la pena mencionar algunas de las más ampliamente compartidas. Desde un planteamiento centrado en el discurso de los medios, Tankard et al. (1999) definen los marcos mediáticos como ideas organizadoras centrales del contenido informativo que proveen un contexto y sugieren qué es un tema por medio del uso de cuatro mecanismos: la selección, el énfasis, la exclusión y la elaboración. Esta definición converge para muchos con el segundo nivel de la agenda, es decir, con el estudio de los atributos de los temas y su transferencia de los medios al público (Ghanem, 1997; McCombs, 2004).

No obstante, la definición de frame más ampliamente compartida por la comunidad científica es atribuible a Robert Entman (1993). Su popularidad, no obstante, parece obedecer más a un cierto ritualismo académico de citación que a una auténtica voluntad por operacionalizar su definición utilizando sus componentes específicos.

\begin{abstract}
To frame is to select some aspects of a perceived reality and make them more salient in a communicating text, in such a way as to promote a particular problem definition, causal interpretation, moral evaluation, and/or treatment recommendation for the item described (Entman, 1993: 52).
\end{abstract}

Otros autores enfatizan, por su parte, los matices socioculturales de los marcos, y los definen como "principios organizadores socialmente compartidos y persistentes en el tiempo, que trabajan a nivel simbólico para estructurar de forma significativa el mundo social" (Reese, 2001: 11). Gamson y Modigliani describen los marcos como ideas centrales organizadoras que dotan de sentido a los acontecimientos relevantes, sugiriendo de qué trata un asunto en cuestión (Gamson y Modigliani, 1989). De hecho, Gamson destaca cómo los marcos expanden su potencial simbólico cuando están en consonancia con los mitos, narraciones y rasgos de la cultura en la que se inscriben. "Not all symbols are equally potent. Certain packages have a natural advantage because their ideas and language resonate with larger cultural themes" (Gamson, 1988: 227).

\title{
2. Framing y agenda setting
}

Uno de los debates teóricos más intensos en el seno de la academia de comunicación nos remite a la controversia en torno a independencia de la perspectiva del framing con respecto a la agenda setting. La mayoría de estudiosos sobre la agenda han reclamado la integración del framing en el modelo de la agenda setting, promoviendo una 
concepción de los marcos restringida y prácticamente equiparable a los atributos de los temas (Ghanem, 1997; Takeshita, 1997; McCombs, 2004; Weaver, 2007). "Framing is the selection of a restricted number of thematic related attributes for inclusion on the media agenda when a particular object is discussed" (McCombs, 1997: 37).

Otros han denunciado esta vocación hegemónica de la agenda setting y criticado los intentos de subsumir el framing bajo su paraguas, señalando que existen diferencias teóricas y epistemológicas que no permiten tal equiparación (Kosicki, 1993; Pan y Kosicki, 1993; Reese, 2001, Maher, 2001). Arguyen que el framing supone precisamente la apertura y superación de las limitaciones de la agenda setting (Kosicki, 1993; Reese, 2001).

The framing perspective is important because it provides a way to view issues that goes beyond a simple researcher-designated label that takes all the controversy out of the issue. [...] In contrast, the current dominant agenda-setting framework strips away almost everything worth knowing about how the media cover an issue and leaves only the shell of the topic (Kosicki, 1993: 112).

Mientras que la agenda setting constituye un modelo unificado, fundamentado sobre una epistemología neopositivista, dotado de procedimientos metodológicos cuantitativos que han avalado empíricamente de forma sucesiva la capacidad de los medios de transferir relevancia temática al público sobre un conjunto de temas (McCombs y Shaw, 1972; Canel et al., 1996), el framing constituye una perspectiva de análisis capaz de dar cuenta de la naturaleza conflictiva y construida de las controversias públicas, de diseccionar el proceso por el cual los asuntos públicos son definidos en uno términos y no en otros, y de abordar, en definitiva, las interacciones simbólicas que subyacen a la producción del discurso mediático y sus efectos sobre las cogniciones individuales (Pan y Kosicki, 1993 y 2001; Maher, 2001; Carragee y Roefs, 2004; Reese, 2007).

Actualmente, la mayoría reconoce la independencia del framing de la agenda setting y ambas perspectivas son consideradas complementarias (Scheufele, 2000; Scheufele y Tewksbury, 2007). No obstante, la importante influencia de los estudios de agenda sobre el desarrollo del framing y las diferencias epistemológicas que subyacen a su origen multidisciplinar ${ }^{4}$ han dado lugar a dos grandes corrientes de investigación. Por una parte, una corriente cuantitativa, que importa la epistemología neopositivista y los métodos del modelo de la agenda setting. Por otra, una corriente interpretativa, heredera de la sociología constructivista y partidaria de explorar la producción del discurso y las representaciones sociales a través de métodos cualitativos.

La corriente cuantitativa ha desarrollado innumerables modelos de análisis de contenido, medición de frecuencias de palabras y análisis por categorías semánticas agrupadas (Tankard, 2001; Miller et al., 1998; Miller y Riechert, 2001; Shah et al., 2001). La mayoría de estas apuestas identifican los encuadres periodísticos por medio de procedimientos estandarizados analizando el contenido manifiesto en busca

${ }^{4}$ Para un análisis detallado en castellano, remitimos a Miceviciute (2013). 
de frame terms, a saber, indicadores de formas específicas de problematización que revelan los puntos de anclaje sobre cuya base se discuten e interpretan los asuntos públicos. Además, prestan atención a la ubicación de los frame terms en dispositivos estratégicos del texto (como los titulares, los subtítulos y los destacados) con el fin de cuantificar su relevancia (Tankard, 1991; Sádaba, 2001).

A priori, estos enfoques permiten no sólo identificar los encuadres periodísticos dominantes, sino también rastrearlos en el discurso de otros actores (como las élites políticas) y analizar sus implicaciones ideológicas. Es decir, permiten el análisis de procesos de frame-sponsorship (Miller et al., 1998; Miller y Riechert, 2001). Sin embargo, este tipo de aproximaciones son notablemente escasas en la literatura internacional (Carragee y Roefs, 2004; Borah, 2011).

Por su parte, la corriente interpretativa hunde sus raíces en una epistemología constructivista y se inclina por técnicas de investigación cualitativas, pues considera que una metodología cuantitativa no puede ofrecer una comprensión suficiente de la construcción del discurso público y mediático (Gamson y Modigliani, 1989; Gamson, 1992; Pan y Kosicki, 1993; Pan y Kosicki, 2001; Reese, 2001; Hertog y McLeod, 2001).

Esta aproximación ha sido especialmente desarrollada por los estudios de los marcos de la acción colectiva, a saber, las estrategas simbólicas que los movimientos sociales despliegan para promover la movilización (Snow et al., 1986; Gamson y Modigliani, 1989; Sampedro, 1996; Benford y Snow, 2000). "Social movement scholars interested in framing processes begin by taking as problematic what until the mid-1980s the literature largely ignored: meaning work- the struggle over the production of mobilizing and countermobilizing ideas and meanings" (Benford y Snow, 2000: 613).

Los marcos de acción colectiva se conciben como las estrategias discursivas que dan sentido a situaciones sociales y permiten que se desarrolle la acción colectiva (Gamson, 1992), ya que "la movilización no sólo requiere que las condiciones estructurales hayan madurado..., también requiere una masa crítica de personas que definan colectivamente la situación como madura y persuadan a otras de que su versión de la realidad suena bien" (Benford, 1993: 199). Los marcos de la acción colectiva se generan como resultado de procesos de comprensión y negociación compartida que se producen entre los activistas implicados en torno a algún fenómeno sobre el cual se aspira a intervenir.

En definitiva, pese a la notable expansión del framing y a la pretendida aceptación de su independencia, no existe un paradigma unificado de análisis, sino más bien dos corrientes diferenciadas, que presentan fundamentos epistemológicos irreconciliables. Así lo recoge Stephen Reese, que captura de una forma particularmente brillante las tensiones subyacentes en el seno de la disciplina:

On one hand, traditional behavioral social science strives for data reduction and parsimony, measuring the accumulation of emphasis in the observed and explicit. Quantification's precise measurement makes it preferred by many scholars, but the most important frame may not be the most frequent. So, on the other hand, the qualitative turn of much framing analysis helps resist 
the reductionistic urge to sort media texts and discourse into containers and count their size or frequency. Indeed, some define frames as an inherently qualitative construct. In this case, one must capture the meanings embedded in the internal relations within texts, which collapsing into reductive measures would obscure. The positivist, behavioral measures of frames based on manifest content don't capture the tensions among expressed elements of meaning, or between what is said and what is left unsaid (Reese, 2001: 8).

\section{El framing en España: el sesgo mediocéntrico}

Ante este panorama internacional, conviene analizar qué orientaciones teórico-metodológicas predominan en la academia de comunicación en España. El objetivo no es realizar una revisión comprensiva de las publicaciones sobre framing editadas en España, como ya hacen otros trabajos ${ }^{5}$ (Vicente y López, 2009; Ardévol-Abreu, 2015), ni tampoco dar cuenta de todas las contribuciones de autores españoles a la disciplina, sino reflexionar críticamente sobre las principales orientaciones teóricometodológicas que prevalecen en los estudios de framing en España.

Se trata, esencialmente, de tres apreciaciones relacionadas: 1) la concepción de los encuadres noticiosos por parte de la mayoría de investigadores españoles como el resultado exclusivo de las rutinas periodísticas, 2) la orientación eminentemente cuantitativa, y 3) la equiparación de gran parte de los estudios de framing con el segundo nivel de la agenda, pese al reconocimiento teórico de su independencia. La exposición de estas tendencias nos permitirá señalar algunas limitaciones, que, a nuestro juicio, restringen la riqueza analítica que ofrece el framing.

\subsection{La concepción de los marcos como resultado de las rutinas periodísticas}

En primer lugar, llama la atención que la mayoría de investigadores españoles manejen una concepción de los marcos mediáticos como resultado exclusivo de las rutinas periodísticas (ver, por ejemplo, Ballesteros, 2015; Muñiz, 2015; Cheng et al., 2010; Berganza, 2008). Los encuadres son concebidos como dispositivos organizadores de los mensajes, ideológicamente neutros y generados sin la concurrencia de influencias extramediáticas. Esta asunción teórica equivale a concebir los encuadres periodísticos como el fruto de una decisión más o menos consciente de los periodistas. Por ejemplo, Sádaba (2001: 159) señala que "la teoría del framing [...] argumenta que, cuando cuenta lo que sucede, el periodista encuadra la realidad y aporta su punto de vista".

Es decir, la investigación española tiende a asumir que la producción de los encuadres periodísticos se desarrolla sin ningún tipo de influencia extramediática, en

\footnotetext{
5 Vicente y López (2009) concluyen, tras una revisión exhaustiva de los estudios que aplican la teoría del encuadre y la realización de entrevistas a expertos, que la inexistencia de grandes equipos de investigación, la falta de coordinación entre centros de trabajo, y los problemas de definición conceptual y operacionalización de los marcos constituyen los principales obstáculos para el despegue definitivo de la disciplina. Por su parte, Ardévol-Abreu (2015) llega a conclusiones similares, pese a detectar un aumento notable del número de publicaciones en los últimos años. "La especialidad parece que no acaba de arrancar... el número de investigadores es reducido y unos pocos nombres se repiten" (p. 440).
} 
consonancia con parte de la academia internacional. "Much news frame analysis implicitly assumes the determining agency of the individual journalist or editor" (Vliegenthart y Zoonen, 2011: 108). La batalla por la construcción de significados entre diversos actores sociales organizados en la arena mediáica queda, por lo tanto, generalmente excluida de la agenda de investigación en España.

En este sentido, algunos autores españoles señalan que existen diferencias en la concepción de encuadres periodísticos, por una parte, y en la de los marcos de acción colectiva o advocate frames, por otra (ver, por ejemplo, Sádaba, 2001 o Miceviciute, 2013). Subrayan que frente a la clara dimensión ideológica de los collective action frames, los marcos mediáticos se sitúan en un plano de organización y estructuración cognitiva de la información: "los encuadres que se utilizan en los medios, en principio, se entiende que surgen como resultado del trabajo periodístico, que son parte de todo acercamiento interpretativo a la realidad y de una serie de circunstancias profesionales" (Sádaba, 2001: 155).

Esta diferenciación, presente en muchos trabajos, resulta, a nuestro juicio, algo artificial, en tanto que el hecho de que los encuadres periodísticos no estén concebidos para la acción política no implica que no respondan a una visión ideológica de la realidad, fundamentada en valores y relaciones de poder (Carragee y Roefs, 2004).

Muchos investigadores españoles reconocen, no obstante, los esfuerzos de los actores sociales para trasladar su marcos a los medios (Sádaba, 2001; Sádaba et al., 2012; Miceviciute, 2013; Árdevol-Abreu, 2015). Sin embargo, este reconocimiento teórico apenas se ha traducido todavía en una vocación empírica por analizar las implicaciones ideológicas de los encuadres periodísticos que dominan los mensajes, ni tampoco por averiguar si éstos reproducen las narrativas ofrecidas por actores políticos o sociales organizados.

A este respecto, creemos que esta concepción de los marcos como producto esencialmente periodístico supone el aislamiento del discurso mediático del contexto político, social y cultural en el que se produce, además de una notable exageración de la autonomía periodística (Carragee y Roefs, 2004). Desde un punto de vista teórico, resulta difícil sostener la idea de que los medios operan en un vacío ideológico. Muy al contrario, los medios constituyen un agente social de primer orden en la definición e interpretación de la realidad (Pan y Kosicki, 2001) pero que opera en un contexto de múltiples influencias sociales y políticas. Para algunos, incluso, los medios reproducen los discursos sociales hegemónicos (Gitlin, 1980; Hall, 1982; Carragee, 1991). "The social and political world is not a pre-given 'hard' reality for the media to reflect, it has to be socially constructed" (Hackett, 1984: 234).

Si aceptamos la idea de que los medios no operan en un vacío ideológico, cabría interrogarse no sólo por los encuadres periodísticos, sino por su origen, rastreándolos en la producción simbólica de actores sociales organizados y analizando sus implicaciones ideológicas (Carragee y Roefs, 2004). Una tarea, sin embargo, que ha resultado generalmente desatendida hasta la fecha por la academia española, con algunas excepciones (Castelló y Monteagut, 2011; Bartolomé y Rodríguez-Virgili, 2012; Carratalá, 2013 y 2014; Valera, 2014).

Los trabajos de Carratalá $(2013,2014)$ analizan la producción simbólica de movimientos conservadores en contra de la producción legislativa de diversos gobiernos progresistas en España (de la Segunda República, por una parte, y del gobierno de 
Zapatero, por otra), y examinan cómo la prensa conservadora adopta estos marcos de acción colectiva en su cobertura informativa. Sus resultados revelan procesos de frame-sponsorship, por los cuales los diarios conservadores adoptan generosamente los encuadres producidos por estos movimientos de protesta. Lo mismo sucede con Valera (2014), que revela cómo la prensa tradicional reproduce los marcos de los partidos políticos ideológicamente afines durante la campaña electoral de 2011, si bien lo hace negociando e incluso confrontando algunos de sus aspectos específicos.

En esta misma línea, Castelló y Monteagut (2011) ilustran cómo la batalla por enmarcar los asuntos públicos constituye una dura competición simbólica entre élites políticas y medios de comunicación. Tras realizar entrevistas a periodistas de radio y asesores de comunicación, concluyen que lejos de adoptar automáticamente los encuadres partidistas, los periodistas de radio negocian hábilmente los significados emitidos por las élites políticas. Su trabajo supone un excelente ejemplo de la aplicación del framing al proceso de construcción de discurso público, que pone el foco en la interacción simbólica entre actores. Por su parte, Bartolomé y Rodríguez-Virgili (2012) analizan si el marco sobre la situación económica promovido por el Gobierno de Zapatero fue capaz de imponerse en el espacio público.

Por el contrario, la mayoría de estudios en España sobre los marcos que estructuran el discurso público en torno a conflictos políticos y sociales de primer orden toman los encuadres periodísticos de estos acontecimientos como dispositivos que emanan directamente de las redacciones periodísticas (Sádaba y Rodríguez Virgili, 2007; Ballesteros, 2015; Muñiz et al., 2008, Igartua, Muñiz y Cheng, 2005). Asumen que quien determina el encuadre de los mensajes es el periodista, descartando de antemano que el discurso producido por los distintos actores sociales implicados en las controversias pudiera tener influencia sobre los contenidos mediáticos. Este sesgo mediocéntrico, en definitiva, tiene como consecuencia que el contenido mediático es tomado de forma cuasi sistemática como variable independiente.

Más allá de esta objeción teórica, existen también razones empíricas que invitan a indagar las implicaciones ideológicas de los marcos mediáticos, y no sólo a concebirlos como el resultado de las rutinas periodísticas. La primera es el pluralismo polarizado del sistema mediático español, que exhibe un alto grado de paralelismo político (Hallin y Mancini, 2004). Otras razones remiten a los estudios que demuestran que los periodistas españoles siguen exhibiendo actitudes sacerdotales hacia la política (Canel, Rodríguez y Sánchez, 2000; Humanes, Martínez y Saperas, 2013), y que la prensa escrita se alinea con los partidos ideológicamente cercanos mediante la reproducción de las agendas partidistas (Valera, 2015). Resulta, por lo tanto, más que pertinente estudiar el origen de los encuadres periodísticos. Así lo reclaman, además, diversas voces internacionales que reivindican el potencial del framing como herramienta de análisis de la interacción simbólica entre actores políticos y mediáticos (Carragee y Roefs, 2004; Borah, 2011).

\subsection{La orientación cuantitativa}

En segundo lugar, creemos conveniente destacar la orientación predominantemente cuantitativa de gran parte de la investigación española sobre framing (Vicente y López, 2009; Ardévol-Abreu, 2015). Muchos frame studies desarrollados en España se 
ubican, de hecho, en la estela marcada por la mencionada corriente cuantitativa, entre los que destaca el equipo liderado por Juan José Igartua, sin duda el más prolífico y estable a lo largo de la última década, y el que ha generado la producción científica de más alto nivel.

Los trabajos realizados por el equipo de Igartua (ver, por ejemplo, Igartua, Muñiz y Cheng, 2005; Cheng et al., 2009; Cheng et al., 2010) han arrojado numerosos resultados sobre los marcos que estructuran el tratamiento informativo de la inmigración. Su aproximación metodológica se sustenta en el análisis de contenido orientado a la medición de atributos en los textos noticiosos (ver, por ejemplo, Muñiz et al., 2009; Cheng et al., 2010), y también en diseños experimentales para dar cuenta de los efectos de los encuadres en el público (Muñiz et al., 2009). Sus trabajos han propiciado una creciente sofisticación metodológica en el estudio del framing en España, dando lugar a modelos notablemente consolidados de análisis (Vicente y López, 2009).

Sin embargo, esta preferencia por el cuantitativismo implica, a nuestro juicio, una serie de limitaciones que coartan el potencial explicativo del framing como herramienta de análisis del discurso mediático. En primer lugar, porque las aproximaciones cuantitativas operan únicamente sobre el contenido manifiesto de los mensajes, de forma que las omisiones estratégicas del discurso pasan inadvertidas en los análisis. Esta objeción, que puede parecer banal, reviste, sin embargo, gran importancia teórica, ya que los frames se definen tanto por los aspectos que enfatizan como por los que silencian. "Most frames are defined by what they omit as well as include, and the omissions of potential problem definitions, explanations, evaluations, and recommendations may be as critical as the inclusion in guiding the audience" (Entman, 1993: 54).

Así, la cuantificación de los encuadres periodísticos que dominan el discurso mediático sólo es relevante en la medida en que otros encuadres son marginados o, directamente, excluidos. Los enfoques cuantitativos ofrecen información precisa y fiable sobre la utilización de marcos específicos, pero son incapaces de responder por todo aquello que está ausente del contenido manifiesto, y, sin embargo, puede ser igualmente decisivo en la problematización de los asuntos públicos. "Qualitative study of frames is inevitable (...) Human judgment is necessary to aroach the essential question about what could have been in the text but was not" (Hertog y McLeod, 2001: 152-153).

En segundo lugar, las aproximaciones cuantitativas dificultan el estudio contextualizado de los marcos mediáticos en los espacios institucionales y culturales en los que se inscriben (Gamson y Modigliani, 1989; Pan y Kosicki, 1993; 2001; Reese, 2001). Así, cuestiones como la resonancia cultural y el potencial simbólico de los marcos quedan desatendidas, y lo mismo ocurre con las condiciones estratégicas específicas en que se despliegan las interacciones simbólicas entre actores (Rucht, 1990). En este sentido, sólo la mirada del investigador puede situar el estudio de los marcos en el contexto cultural más amplio en el que éstos son producidos (Gamson; 1988; Hertog y McLeod, 2001; Carragee y Roefs, 2004). En España, sin embargo, sólo algunos estudios incluyen la contextualización cultural en el análisis de los encuadres periodísticos (ver, por ejemplo, Sádaba, 2004; Zamora y Marín, 2010).

Además, en esta corriente cuantitativa que domina la producción científica en España, hallamos estudios afanados por el rastreo sistemático de frames previamente 
establecidos por la literatura científica, que se conciben como patrones universales de la cobertura periodística y que funcionan como garantían de cierta objetividad informativa (Miceviciute, 2013). Esta "vía deductiva" (Ardévol-Abreu, 2015) goza de especial predicamento en la investigación europea (Semetko y Valkenburg, 2000) y ha sido también adoptada por la academia española (ver, por ejemplo, Sádaba y Rodríguez-Virgili, 2007; Castelló y Capdevila, 2013; Sánchez-Castillo, 2011; Sánchez-Castillo y Mercado Sáez, 2013).

Algunos de estos marcos predefinidos son los encuadres temáticos o estratégicos aplicados a la cobertura de campañas electorales (Muñiz, 2015; Berganza et al., 2011; Berganza, 2008), y también los llamados generic frames (consecuencias económicas, conflicto, interés humano, atribución de responsabilidad y moralidad), a saber, encuadres habituales de cobertura mediática (Valkenburg, Semetko y De Vreese, 1999; Semetko y Valkenburg, 2000).

Pese a que esta vía deductiva facilita los estudios comparativos y favorece la replicabilidad, creemos conveniente señalar que estas categorizaciones presentan también problemas de ambigüedad conceptual, puesto que no se derivan de una clara y única definición de frame. Más bien, los distintos tipos de marcos genéricos se ocupan de aspectos no necesariamente equiparables, sino complementarios, del proceso de enmarcado, como son los mecanismos de explicación causal (la atribución de responsabilidades) o la problematización de lo que está en juego (moralidad, consecuencias económicas, interés humano). Cada uno de estos encuadres se ocupa, de hecho, de componentes específicos del framing, según la definición más ampliamente compartida por la academia internacional (Entman, 1993). Pero además, estas aproximaciones pueden resultar reduccionistas por cuanto excluyen del análisis todos los posibles marcos (más allá de estas categorizaciones predefinidas) que pudieran estar presentes en el discurso mediático (Igartua y Humanes, 2004).

\subsection{El difícil divorcio de la agenda setting}

Finalmente, conviene señalar el complicado proceso de emancipación que sigue experimentado el framing con respecto al modelo de la agenda setting. Ello se debe a que la mayoría de estudios descansan sobre definiciones operativas de los encuadres como conjuntos de atributos temáticamente relacionados (ver, por ejemplo, Ballesteros, 2015; Cheng et al., 2009; Cheng et al., 2010; Muñiz et al., 2009), es decir, despliegan una operacionalización equiparable a la propuesta por los analistas del segundo nivel de la agenda. Así, pese a que muchos autores españoles reclaman la independencia del framing como paradigma de investigación, sus aproximaciones específicas de análisis exhiben una notoria dependencia de los presupuestos teóricometodológicos del modelo de la agenda setting.

Consideramos oportuno señalar que para completar la emancipación del framing de su predecesor, resultaría necesario diversificar las definiciones operativas de los marcos más allá de los conjuntos de atributos temáticamente relacionados. En este sentido, Entman (1993) ofrece un buen punto de partida para la operacionalización de los marcos como la combinación de cuatro operaciones discursivas (problematización, interpretación causal y atribución de responsabilidades, evaluación moral y propuestas de solución), que además converge con las tareas diagnóstica y prognóstica que los 
estudiosos de los movimientos sociales atribuyen a los marcos de acción colectiva (Benford y Snow, 2000). El valor de estas definiciones reside precisamente en que conciben los marcos como estructuras subyacentes a la producción simbólica de cualquier actor social.

Creemos que esta diversificación de los métodos de identificación y medición de los marcos supondría explotar más intensamente la riqueza analítica de este paradigma de investigación para dar cuenta de los complejos procesos de construcción y negociación del discurso público, tal como demuestran algunos estudios (Sádaba, 2004; Carratalá, 2013; Valera, 2014). Porque el framing permite precisamente ir más allá de la medición de atributos temáticos para dar cuenta de cómo se definen persuasivamente las controversias por parte de diversos actores sociales, y qué interpretaciones de la realidad se imponen en el discurso mediático.

\begin{abstract}
[framing] expands beyond what people talk or think about by examining how they think and talk (...) The crucial advance from the agenda-setting research is that framing analysis examines the diversity and fluidity in how issues are conceptualized and consequently allows for more fruitful analysis of the conceptual evolution of policy issues (Pan y Kosicki, 1993: 70).
\end{abstract}

\title{
4. Conclusiones
}

Tras esta revisión crítica de los enfoques teórico-metodológicos predominantes en los estudios sobre framing en España, proponemos, a modo de conclusión, dos recomendaciones orientadas a superar el sesgo mediocéntrico: a) recuperar el anclaje sociológico de la disciplina, situando los estudios sobre los marcos mediáticos en el contexto social en el que se producen, atendiendo a las múltiples influencias que operan en la producción de los encuadres periodísticos, y b) diversificar los métodos de investigación, aprovechando la riqueza que ofrecen los métodos cualitativos para dar cuenta de las implicaciones ideológicas del framing y de sus resonancias culturales.

En primer lugar, consideramos pertinente recuperar el anclaje sociológico de la disciplina, complementando el estudio de la descripción de los encuadres mediáticos y sus efectos con el análisis de la producción y negociación simbólica entre diversos actores (Vliegenthart y Zoonen, 2011; Borah, 2011; Carragee y Roefs, 2004), como ya sucede de forma minoritaria (Castelló y Monteagut, 2011; Carratalá, 2013 y 2014). Así lo ha hecho ya parte de la academia internacional, ocupándose de rastrear la influencia de los actores políticos en la génesis de los marcos mediáticos, e indagando cómo las definiciones de las controversias públicas promovidas por los actores políticos influyen en la cobertura informativa (Hänggli, 2011; Hänggli y Kriesi, 2012).

Creemos que el enorme interés que revisten los estudios aquí citados se expandiría si también contemplaran el análisis del origen de los marcos, pues ello permitiría explotar plenamente el potencial explicativo del framing para el estudio de la comunicación política (Sádaba et al., 2012). Porque la construcción de los encuadres mediáticos no es una operación exclusiva ni genuinamente periodística, ni tampoco 
puede conceptualizarse como una tabula rasa desde el punto de vista ideológico. ¿Cómo, si no, explicar el hecho de que algunas interpretaciones de la realidad consigan penetrar en el discurso mediático y otras no lo hagan?

En segundo lugar, proponemos también una diversificación de los métodos de investigación y las estrategias de operacionalización, con especial atención a los métodos cualitativos. El análisis del discurso resulta, en este sentido, irrenunciable para dilucidar cómo diversos actores organizados construyen selectivamente la realidad en contextos específicos, cómo enfatizan y omiten aspectos cruciales en sus interpretaciones y cómo estos significados son reproducidos y/o negociados en el discurso mediático (Entman, 1993; Pan y Kosicki, 1993; 2001; Reese, 2001; Carragee y Roefs, 2004). También el uso de técnicas como las entrevistas en profundidad enriquecerían el estudio de la producción discursiva e interacción simbólica entre actores, como ya han demostrado algunos trabajos (Castelló y Monteagut, 2011).

Ello redundaría, en definitiva, en la emancipación definitiva del framing de la agenda setting, y en su consolidación como un modelo de investigación capaz no sólo de determinar los encuadres periodísticos y sus efectos sobre el público, sino también de dar cuenta de la naturaleza construida y conflictiva del discurso público, situando el estudio de los mensajes periodísticos en el contexto de las múltiples disputas ideológicas que se producen en el espacio público.

\section{Referencias bibliográficas}

ARDÉVOL-ABREU, A. (2015). Framing o teoría del encuadre en comunicación: Orígenes, desarrollo y panorama actual en España. Revista Latina de Comunicación Social, 70, 423-450. http://dx.doi.org/10.4185/RLCS-2015-1053

BALLESTEROS, C. (2015). Naciones mediáticas: Los marcos informativos de la consulta sobre la independencia de Cataluña (9-N). Doxa Comunicación, 20, 161-183.

BARTOLOMÉ Castro, J.M. y RODRÍGUEZ-VIRGILI, J. (2012). Pugna de frames en el debate político español sobre la situación económica (2008-2011). Estudios sobre el Mensaje Periodístico, 18(2), 439-457. http://dx.doi.org/10.5209/ rev_ESMP.2012.v18.n2.41018

BATESON, G. (1972). Steps to an Ecology of Mind. Collected Essays in Antropology, Psychiatry, Evolution and Epistemology. Nueva York. Ballantine Books.

BENDFORD, R. (1993). Frame disputes within the nuclear disarmament movement. Social Forces, 37, 677-701. http://dx.doi.org/10.1093/sf/71.3.677

BENFORD, R. y Snow, D. (2000). Framing processes and social movements: An overview and assessment. Annual Review of Sociology, 26, 611-639. http://dx. doi.org/10.1146/annurev.soc.26.1.611

BERGANZA, R. (2008). Medios de comunicación, 'espiral del silencio' y desconfianza política: Estudio de caso de la cobertura mediática de los comicios electorales europeos. Zer. Revista de Estudios de Comnicación, 25, 121-139.

BERGANZA, R., DE MIGUEL, R. y CHAPARRO, M. A. (2011). La prensa gratuita ante las elecciones generales de España del 9 de marzo de 2008: cobertura, 
politización y frames en contraposición a la prensa de pago. Comunicación y Sociedad, 24, 35-61.

BORAH, P. (2011). Conceptual issues in framing theory: A systematic examination of a decade's literature. Journal of Communication, 61, 246-263.

http://dx.doi.org/10.1111/j.1460-2466.2011.01539.x

CANEL, M. J., LLAMAS, J. P. y REY, F. (1996). El primer nivel del efecto agendasetting en la información local: los problemas más importantes de la ciudad de Pamplona. Comunicación y Sociedad, 9, 17-38.

CANEL, M. J., RODRÍGUEZ, R. y SÁNCHEZ, J. J . (2000). Periodistas al descubierto. Retrato de los profesionales de la información. Madrid: CIS.

CARRAGEE, K. (1991). News and Ideology: An Analysis and Coverage of the West German Green Party by The New York Times. Journalism Monographs, 128, 1-30.

CARRAGEE, K. y ROEFS, W. (2004). The Neglect of Power in Recent Framing Research. Journal of Communication, 54(2), 214-233. http://dx.doi. org/10.1111/j.1460-2466.2004.tb02625.x

CARRATALÁ, A. (2013). Los marcos de acción colectiva en la prensa conservadora. Observatorio Journal, 7(1), 133-171.

CARRATALÁ,A. (2014). Voces católicas y propaganda movilizadora ante la Ley de Confesiones y Congregaciones Religiosas. Historia y Comunicación Social, 19, 289-299. http://dx.doi.org/10.5209/rev_hics.2014.v19.45133

CASTELLÓ, E. y MONTEAGUT, M. (2011). Journalists, Reframing and Party Public Relations Consultants. Journalism Studies, 12(4), 506-521.

CASTELlÓ, E. y CAPDEVILA, A. (2013). Defining pragmatic and symbolic frames. Newspapers about the Independence during the Scottish and Catalan elections. Estudios sobre el Mensaje Periodístico, 19, 979-999.

CHENG, L., IGARTUA, J. J., PALACIOS, E., ACOSTA, T., OTERO, J. A. y FRUTOS, J. (2009). Aversión vs. aceptación, dos caras de la misma moneda: un estudio empírico de encuadres noticiosos sobre inmigración en la prensa regional de Castilla y León. Zer. Revista de Estudios de Comunicación, 14, 35-57.

CHENG, L., IGARTUA, J. J., PALACIOS, E., ACOSTA, T. y PALITO, S. (2010). Framing Immigration News in Spanish Regional Press. International Migration, $56,197-215$.

D’ANGELO, P. (2002). News Framing as a Multiparadigmatic Research Program. A response to Entman. Journal of Communication, 52, 870-888. http://dx.doi. org/10.1111/j.1460-2466.2002.tb02578.x

ENTMAN,R.(1993).Framing: Towards a Clarification of a Fractured Paradigm.Journal of Communication, 43, 51-58. http://dx.doi.org/10.1111/j.1460-2466.1993. tb01304.x

GAMSON, W. (1988). Political Discourse and Collective Action. International Social Movement Research, 1, 219-244.

GAMSON, W. (1992). Talking Politics. Cambridge. Cambridge University Press.

GAMSON, W. y MODLIGIANI, A. (1989). Media Discourse and Public Opinion on Nuclear Power: A Constructionist Aroach. American Journal of Sociology, 95(1), 1-37. http://dx.doi.org/10.1086/229213

GHANEM, S. (1997). Filling the Tapestry: The Second Level of Agenda Setting. En M. McCombs, D. Shaw y D. Weaver (eds.), Communication and Democracy: 
Exploring the Intellectual Frontiers in Agenda-Setting Theory (pp. 3-14). New Jersey: Lawrence Erlbaum.

GITLIN, T. (1980). The whole world is watching. Mass media in the making and unmaking of the New Left. Berkeley: University of California Press.

GOFFMAN, E. (1986). Frame Analysis. An Essay on the Organization of Experience. Boston: Northeastern University Press.

HACKETT, R. (1984). Decline of a Paradigm? Critical Studies in Mass Communication, 1, 229-259. http://dx.doi.org/10.1080/15295038409360036

HALL, S. (1982). The rediscovery of 'ideology': Return to the repressed in media studies. En M. Gurevitch. (eds.). Culture, society and the media (pp. 56-90). London: Methuen.

HALLIN, D., y MANCINI, P. (2004). Sistemas mediáticos comparados: Tres modelos de relación entre los medios de comunicación y la política. Barcelona: Hacer.

HÄNGGLI, R. (2011). Key Factors in Frame Building. How Strategic Political Actors Shape News Coverage. American Behavioral Scientist, 56, 300-317. http:// dx.doi.org/10.1057/9780230343214_9

HÄNGGLI, R. y KRIESI, H. (2010). Political framing strategies and their impact on media framing in a Swiss direct-democratic campaign. Political Communication, 27, 141-157. http://dx.doi.org/10.1080/10584600903501484

HERTOG, J. y MCLEOD, D. (2001). A multiperspectival Aproach to Framing Analysis. A Field Guide. En S. Reese, O. Gandy y A. Grant (eds.), Framing Public Life. Perspectives on Media and Our Understanding of the Social World (pp. 139-161). Nueva Jersey: Lawrence Erlbaum.

HUMANES, M. L., MARTÍNEZ-NICOLÁS, M. y SAPERAS, E. (2013). Political Journalism in Spain: Practices, Roles and Attitudes. Estudios del Mensaje Periodístico, 19, 715-731.

IGARTUA, J. J., MULIZ, C. y CHENG, L. (2005). La inmigración en la prensa española: Aportaciones empíricas y metodológicas desde la teoría del encuadre noticioso. Migraciones, 17, 143-181.

IGARTUA, J. J. y HUMANES, M. L. (2004). Teoría e investigación en comunicación social. Madrid: Síntesis.

KAHNEMAN, D. y TVERSKY, A. (1979). Prospect theory: Analysis of decision under risk. Econometrica, 47, 263-291. http://dx.doi.org/10.2307/1914185

KOSICKI, G. (1993). Problems and Oortunities in Agenda-Setting Research. Journal of Communication, 43, 100-127. http://dx .doi.org/10.1111/j.1460-2466.1993. tb01265.x

LAKOFF, G. (2004). Don't think of an elephant! Know your values and frame the debate. White River Junction, VT: Chelsea Green.

MAHER, M. (2001). Framing: An Emerging Paradigm or a Phase of Agenda Setting? En S. Reese, O. Gandy y A. Grant (eds.), Framing Public Life. Perspectives on Media and Our Understanding of the Social World (pp. 83-94). Nueva Jersey: Lawrence Erlbaum.

MCCOMBS, M. (1997). News Frontiers in Agenda Setting: Agendas of Attributes and Frames. Mass Comm Review, 24, 32-52.

MCCOMBS, M. (2004). Setting the Agenda: The Mass Media and Public Opinion. Cambridge. Polity Press. 
MCCOMBS, M. y SHAW, D. (1972). The Agenda Setting Function of the Mass Media. Public Opinion Quaterly, 36, 176-187. http://dx.doi.org/10.1086/267990

MICEVICIUTE, J. (2013). Frame periodístico: un concepto puente entre la psicología, la sociología y la linguiística. Zer. Revista de Estudios de Comunicación, 18(35), 71-96.

MILLER, M., ANDSAGER, J. y RIECHERT, B. (1998). Framing the candidates in presidential primaries: issues and images in press releases and news coverage. Journalism \& Mass Communication Quaterly, 75, 312-324. http://dx.doi. org/10.1177/107769909807500207

MILLER, M. y RIECHERT, B. (2001). The Spiral of Opportunity and Frame Resonance: Mapping the Issue Cycle in News and Public Discourse. En S. Reese, O. Gandy y A. Grant (eds.), Framing Public Life. Perspectives on Media and Our Understanding of the Social World (pp. 107-121). Nueva Jersey: Lawrence Erlbaum.

MUÑIZ, C. (2015). La política como debate temático o estratégico. Framing de la campaña electoral mexicana en la prensa digital. Comunicación y Sociedad, 23, 67-95.

MUÑIZ, C., IGARTUA, J. J., DE LA FUENTE, M. y OTERO, J. A. (2008). Imágenes periodísticas de la inmigración: Aportaciones metodológicas al estudio de la comunicación visual. Anàlisi. Quaderns de la Comunicació i Cultura, 37, 31-48.

MUÑIZ, C., IGARTUA, J. J., DE LA FUENTE, M. y OTERO, J. A. (2009). Narrando la inmigración: análisis del tratamiento informativo y evaluación de los efectos de las noticias en España. Revista Mexicana de Ciencias Políticas y Sociales, 207, 117-136.

PAN, Z. y KOSICKI, G. (1993). Framing Analysis: An Approach to News Discourse. Political Communication, 10(1), 55-75. http://dx.doi.org/10.1080/1058 4609.1993.9962963

PAN, Z. y KOSICKI, G. (2001). Framing as a Strategic Action in Public Deliberation. En S. Reese, O. Gandy y A. Grant (eds.), Framing Public Life. Perspectives on Media and Our Understanding of the Social World (pp. 35-65). Nueva Jersey: Lawrence Erlbaum.

REESE, S. (2001). Framing Public Life: A Brigding Model for Media Research. En S. Reese, O. Gandy y A. Grant (eds.), Framing Public Life. Perspectives on Media and Our Understanding of the Social World (pp. 7-31). Nueva Jersey: Lawrence Erlbaum.

REESE, S. (2007). The Framing Project: A Bridging Model for Media Research Revisited. Journal of Communication, 57, 148-154. http://dx.doi. org/10.1111/j.1460-2466.2006.00334.x

RUCHT, D. (1990). Campaign, skirmishes and battles. Anti-Nuclear movements in the USA, France and West Germany. Industrial Crisis Quarterly, 4(3), 193-222. http://dx.doi.org/10.1177/108602669000400304

SÁDABA, T. (2001). Orígenes, aplicación y límites de la 'teoría del encuadre' (framing) en comunicación. Comunicación y Sociedad, 14(2), 143-175.

SÁDABA, T. (2004). Enfoques periodísticos y marcos de participación política: Una aproximación conjunta a la teoría del encuadre. Política y Sociedad, 41, 65-76. 
SÁDABA, T. y RODRÍGUEZ-VIRGILI, J. (2007). La construcción de la agenda de los medios: el debate del Estatut en la prensa española. Ámbitos, 16, 187-211.

SÁDABA, T., RODRÍGUEZ-VIRGILI, J. y BARTOLOMÉ, M. (2012). Propuesta de sistematización de la teoría del framing para el estudio y praxis de la comunicación política. Observatorio Journal, 6(2), 109-126.

SAMPEDRO, V.(1996). Nuevos movimientos sociales, agendas políticas e informativas: el caso de la objeción de conciencia. Madrid: Centro de Estudios Avanzados en Ciencias Sociales.

SÁNCHEZ, S. (2011). Encuadres noticiosos de las enfermedades raras en la prensa española: Perspectiva bajo el análisis de contenido y teoría del framing. En J. FORNIELES y A. BAÑÓN (eds.). Lenguaje, comunicación y salud (pp. 179200). Sevilla: Arcibel.

SÁNCHEZ, S. y MERCADO, M. (2013). Encuadres noticiosos latentes bajo el paradigma positivista: propuesta de análisis factorial exploratorio. Actas del II Congreso Nacional sobre Metodología de la Investigación en Comunicación (pp. 277-294). Segovia: UVA.

SCHEUFELE, D. (2000). Agenda-setting, priming and framing revisited: Another look at cognitive effects of political communication. Mass Communication \& Society, 3, 297-316. http://dx.doi.org/10.1207/s15327825mcs0323_07

SCHEUFELE, D. y TEWKSBURY, D. (2007). Framing, Agenda Setting and Priming: The Evolution of Three Media Effects Models. Journal of Communication, 57, 9-20. http://dx.doi.org/10.1111/j.1460-2466.2006.00326.x

SEMETKO, H. y VALKENBURG, P. (2000). Framing European politics: a content analysis of press and television news. Journal of Communication, 50, 93-109. http://dx.doi.org/10.1111/j.1460-2466.2000.tb02843.x

SHAH, D., DOMKE, D. y WACKMAN, D. (2001). The Effects of Value-Framing on Political Judgment and Reasoning. En S. Reese, O. Gandy y A. Grant (eds.), Framing Public Life. Perspectives on Media and Our Understanding of the Social World (pp. 227-244). Nueva Jersey: Lawrence Erlbaum.

SNOW, D. et al. (1986). Frame Alignment Processes: Micromobilization and Movement Participation. American Sociological Review, 51, 464-481. http://dx.doi. org/10.2307/2095581

TAKESHITA, T. (1997). Exploring the Media's Roles in Defining Reality: From Issue-Agenda Setting to Attribute-Agenda Setting. En M. McCombs, D. Shaw y D. Weaver (eds.), Communication and Democracy: Exploring the Intellectual Frontiers in Agenda-Setting Theory (pp. 15-27). New Jersey: Lawrence Erlbaum.

TANKARD, J. (2001). The Empirical Aroach to the Study of Media Framing. En S. Reese, O. Gandy y A. Grant (eds.), Framing Public Life. Perspectives on Media and Our Understanding of the Social World (pp. 95-106). Nueva Jersey: Lawrence Erlbaum.

TANKARD, J. et al. (1991). Media frames: Aroaches to Conceptualization and Measurement. Presentado en la Association for Education in Journalism and Mass Communication, 1991, Boston.

TUCHMAN, G. (1978). Making news. A study in the construction of reality. New York: Free Press. 
VALERA Ordaz, L. (2014). Agenda building y frame promotion en la campaña electoral de 2011: la circulación del discurso entre partidos, medios y ciudadanos. Tesis doctoral no publicada. Universidad de Valencia.

VALERA Ordaz, L. (2015). Building the news media agenda: Spanish parties' influence on traditional media and blogosphere agendas during 2011 electoral campaign. Communication \& Society, 28, 115-134. http://dx.doi. org/10.15581/003.28.3.115-134

VALKENBURG, P., Semetko, H. y De Vreese, C. (1999). The Effects of News Frames on Readers' Thoughts and Recall. Communication Research, 26, 550569. http://dx.doi.org/10.1177/009365099026005002

VAN GORP, B. (2007). The Constructionist Approach to Framing: Bringing Culture Back in. Journal of Communication, 57, 60-78. http://dx.doi. org/10.1111/j.1460-2466.2006.00329.x

VICENTE, M.; LÓPEZ-RABADÁN, P. (2009). Resultados actuales de la investigación sobre framing: sólido avance internacional y arranque de la especialidad en España. Zer. Revista de Estudios de Comunicación, 14(26), 13-34.

VLIEGENTHART, R. y VAN ZOONEN, L. (2011). Power to the frame: Bringing sociology back to frame analysis. European Journal of Communication, 26(2), 101-115. http://dx.doi.org/10.1177/0267323111404838

WEAVER, D. (2007). Thoughts on Agenda Setting, Framing and Priming. Journal of Communication, 57, 142-147. http://dx.doi. org/10.1111/j.1460-2466.2006.00333.x

ZAMORA, R. y MARÍN, J. A. (2010). La representación simbólica del escándalo político: hacia una tipología de los marcos periodísticos (frames) utilizados en la narración del escándalo de corrupción política. Razón y Palabra, 73. 